\title{
Forecasting the bladder tumor size and immune response of a patient over the time using a dynamic mathematical model
}

\author{
Clara Burgos $^{1}$, Noemí García-Medina ${ }^{1}$, \\ David Martínez-Rodríguez ${ }^{1}$, José-Luis Pontones ${ }^{2}$, \\ David Ramos ${ }^{3}$, Rafael-J. Villanueva ${ }^{1}$ \\ ${ }^{1}$ Instituto Universitario de Matemática Multidisciplinar, \\ Universitat Politècnica de València, Valencia, Spain \\ 2 Department of Urology, \\ Hospital Universitari i Politècnic La Fe, Valencia, Spain \\ 3 Department of Pathology, \\ Hospital Universitari i Politècnic La Fe, Valencia, Spain \\ email: clabursi@posgrado.upv.es, nogarme@alumni.uv.es, \\ damarro3@etsii.upv.es, pontones_jos@gva.es, \\ david.ramos@uv.es, rjvillan@imm.upv.es
}

May 30, 2018

\begin{abstract}
Bladder cancer is one of the most common malignant diseases in the urinary system and a highly aggressive neoplasm. The prognosis is not favourable usually and its evolution for particular patients is very difficult to find out. In this paper we propose a dynamic mathematical model that describes the bladder tumor growth and the immune response evolution. This model is customized for a single patient, determining appropriate model parameter values via model calibration. Due to the uncertainty of the tumor evolution, using the calibrated model parameters, we predict the tumor size and the immune response evolution over the next few months assuming three different scenarios: favourable, neutral and unfavourable. In the former, the cancer disappears; in the second a $5 \mathrm{~mm}$ tumor is expected around the middle of August 2018; in the worst scenario, a $5 \mathrm{~mm}$ tumor is expected around the end of May 2018. The patient has been cited around June 15th, 2018, to check the tumor size, if it exists.
\end{abstract}


Keywords: Bladder Cancer, Dynamic Model, Uncertainty Quantification, Model Calibration, Cancer Prognosis.

\section{Introduction}

Bladder cancer is one of the most common malignant diseases in the urinary system and a highly aggressive neoplasm $[2,5]$. Around $80 \%$ of patients diagnosed with this cancer present a non invasive carcinoma that can be handled via a Trans Urethral Resection (TUR), a surgical endoscopic procedure to remove the tumor from the inner part of the bladder [4]. The bladder cancer is characterized by recursiveness for more than a half of the patients: the tumor appears again after a while and may progress to become a muscle invasive cancer. It is then, when the treatments turn more aggressive, including the removal of the bladder to avoid the spread of the cancer to other parts of the body [6].

The prognosis of bladder cancer is not generally good and after the TUR, the typical treatment consist of instillations of Bacillus Calmette-Guérin (BCG) into the bladder, with the aim that the BCG stimulates the patient's immune response against the cancer and then, the cancer cells may be eliminated [8].

Here, we propose a mathematical model to study the evolution of the bladder cancer of a patient where TURs and the administration of BCG have been considered in these evolution. To our knowledge, only in the paper [3], the authors introduce this kind of model using average parameter values obtained from several in silico patients data.

At this point, we must say that, even though our starting point is [3], we have to adapt the model to be applied with the available data of our patient, given that the available data are those the pathologist considers relevant to be analyzed in order to determine the evolution of the sickness rather than the data necessary for the model.

Thus, we are going to propose a model to describe the evolution of the bladder cancer, we will use data of a patient to determine the model parameters that describe the known evolution and use these model parameters to predict the evolution of the bladder cancer in the short-term. Due to the lack of data, we will perform the evolution taking into account three possible scenarios: favourable, neutral and unfavourable.

The paper is organized as follows. In Section 2, we will introduce the data available and the adaption of the model presented in [3]. In Section 3, we find the model parameter values that allow the model to describe the bladder cancer evolution until now. In Section 4, we predict the evolution of the size of the cancer over the next few months in three scenarios: favourable, neutral and unfavourable. Finally, we present some conclusions of the work.

\section{Available data and model building}

Typically, in the Spanish Region of Valencia, a patient goes to the family doctor or the hospital emergencies when he/she starts to urinate blood (hematuria). 
Then, the patient is addressed to the urologist, who, using ultrasound images or a cytoscopy, diagnoses if there is a tumor. If so, the patient is treated by the doctors in the Urology Department. In our case, we are working with doctors belonging to the Hospital Universitari i Politècnic La Fe [1] in Valencia, Spain. The doctors have collected biological tissues of patients suffering pT1G3 bladder cancer, a type of superficial bladder cancer. The usual protocol is to perform a TUR to the patient, then instillations of BCG and cytologys and cystoscopy for follow-up control and revisions. In case of recurrence, the doctors repeat the cycle describe above until the patient is completely healthy or the stage of the cancer increases and more aggressive treatments have to be applied.

Among the patients, we have chosen from the Hospital Universitari i Politècnic La Fe database one with the complete medical history for our purpose. We are going to call this patient Patient $\mathrm{X}$, and the available data and dates were collected and summarized in Table 1.

\begin{tabular}{cccc}
\hline Date & Activity & Tumor size & Inflammatory cells/field \\
\hline 01/Mar/2012 & Ultrasound & $3-5 \mathrm{~mm}$ & - \\
14/Jun/2012 & TUR & $25 \mathrm{~mm}$ & 260 \\
15/Feb/2015 & Cystoscopy & $1-2 \mathrm{~mm}$ & - \\
28/Apr/2015 & TUR & $5 \mathrm{~mm}$ & 515 \\
30/Jan/2017 & Cystoscopy & $20 \mathrm{~mm}$ & - \\
14/Mar/2017 & TUR & $30-35 \mathrm{~mm}$ & 508 \\
\hline
\end{tabular}

Table 1: Data corresponding to Patient X, who had the first symptoms in March 2012. Since then, the Patient X has suffered three TURs and has been treated with three BCG instillation sessions, each one after each TUR.

Besides the information provided in Table 1, instillation dates are also known. BCG instillations were made the following days: 10/Aug/2012, 26/May/2015 and $27 / \mathrm{Apr} / 2017$, after each TUR. Doses introduced in the bladder consisted of $81 \mathrm{ml}$ of BCG colonies instilled weekly during six weeks.

From paper [3], we have adapted the model there to our available data and to describe properly the clinic practise in the Hospital Universitari i Politècnic La Fe. First, let us denote $E(t)$ as the average inflammatory cells in a microscopy field of view, $T_{u}(t)$ as the radius (size) of the tumor cells non infected by BCG, $T_{i}(t)$ as the radius (size) of the tumor cells infected by BCG, $B(t)$ as the milliliters of BCG inside the bladder and $b(t)$ as the milliliters of BCG injected in one or several doses at the time instant $t$.

Thus, in the first stage, the tumor is growing, no treatment is administered until TUR is performed and only inflammatory cells may interact with the tumor. The tumor growth is modeled by $T_{u}(t+1)=T_{u}(t)+k T_{u}(t)$, where $k$ is the tumor growth rate (exponential growth). Inflammatory cells kill tumor cells at rate $p_{6}$ and it is modeled by the term $-p_{6} E(t) T_{u}(t)$ (Figure 1). Parameter $p_{6}$ measures the effectiveness of the immune system to kill tumoral cells. Also, the production of inflammatory cells increases in presence of tumoral cells and it is modeled by the term $\alpha T_{u}(t)$, where $\alpha$ is the inflammatory cells production 


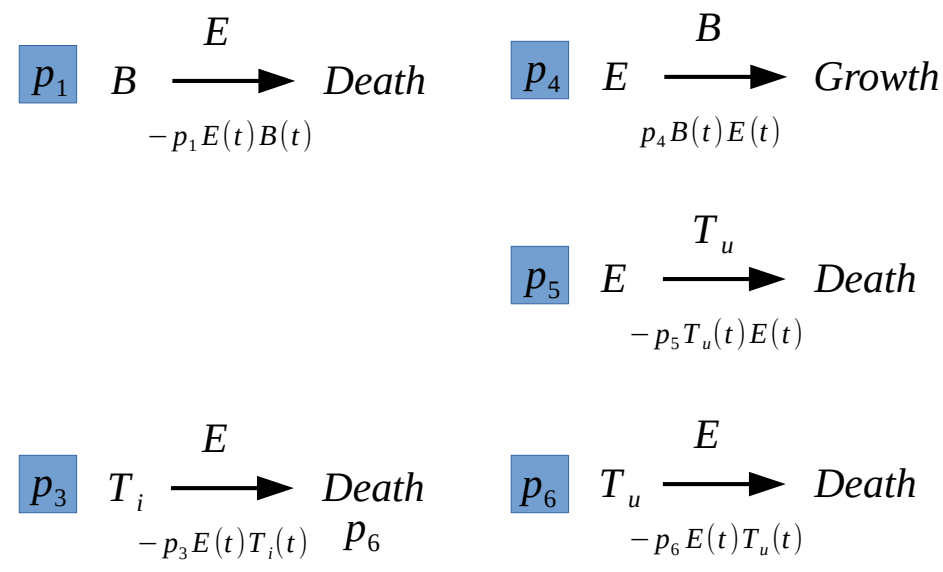

Figure 1: Interaction between BCG $B(t)$, inflammatory cells $E(t)$, tumoral cells $T_{u}(t)$ and tumoral cells infected by BCG $T_{i}(t)$, and their corresponding rates $p_{1}, p_{3}, p_{4}, p_{5}$ and $p_{6}$.

rate.

Then, TUR removes the tumors and after a while, BCG treatment is administered. The tumor is still growing because, although the doctors do their best, the complete removal is almost impossible [9]. But now, tumoral cells are infected by BCG and attacked by the immune system in a more aggressive way than the attack to non-infected cells. Here, infected tumoral cells and BCG have been introduced and we have to describe how they interact. First, the terms $-\mu_{1} B(t)$ and $-\mu_{2} E(t)$ describe the natural deaths of BCG and inflammatory cells, respectively, being $\mu_{1}$ and $\mu_{2}$ their death rates. Now, the interactions (Figure 1):

- the death of BCG because their interaction with inflammatory cells is modeled by $-p_{1} E(t) B(t)$;

- the death of infected tumor cells because of inflammatory cells is modeled by $-p_{3} E(t) T_{i}(t)$;

- the growth of inflammatory cells because of BCG is modeled by $p_{4} B(t) E(t)$;

- the death of inflammatory cells because of uninfected tumor cells is modeled by $-p_{5} T_{u}(t) E(t)$.

The last term to be modeled is the interaction between tumoral cells in presence of BCG (Figure 2). In this case, the tumoral cells become infected at rate $p 2$, and the involved bacillus of BCG die. The term modeling this interaction is given by $p_{2} B(t) T_{u}(t)$ and affects negatively the tumoral cells and BCG and positively the infected tumoral cells. 


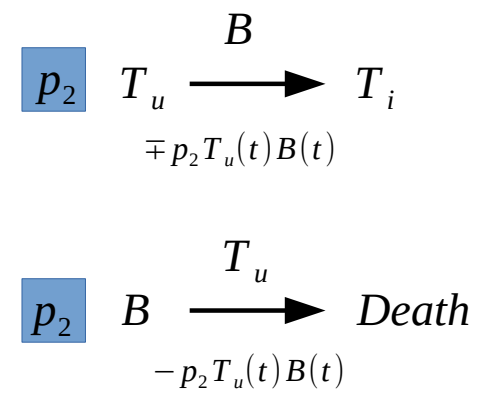

Figure 2: Interaction between BCG $B(t)$ and tumoral cells $T_{u}(t)$, with the corresponding rate $p_{2}$.

Then, gathering all the above terms, the evolution of the dynamics of the tumoral cells, inflammatory cells and BCG can be modeled using the following system of difference equations:

$$
\begin{aligned}
E(t+1) & =E(t)-\mu_{2} E(t)+\alpha T_{u}(t)+p_{4} B(t) E(t)-p_{5} T_{u}(t) E(t), \\
T_{u}(t+1) & =T_{u}(t)-p_{2} T_{u}(t) B(t)-p_{6} E(t) T_{u}(t)+k T_{u}(t) \\
T_{i}(t+1) & =T_{i}(t)-p_{3} E(t) T_{i}(t)+p_{2} T_{u}(t) B(t) \\
B(t+1) & =B(t)-\mu_{1} B(t)-p_{1} E(t) B(t)-p_{2} T_{u}(t) B(t)+b(t)
\end{aligned}
$$

where the parameters have been collected in Table 2 .

\section{Model calibration}

In order to find the model parameters that make the model to be as close as possible to the data of Table 1 in the corresponding time instants, we define the following fitting function $F$ :

INPUT: Model parameter values $\left(k, \alpha, \mu_{1}, \mu_{2}, p_{1}, p_{2}, p_{3}, p_{4}, p_{5}, p_{6}\right)$.

Step 1. Substitute the model parameter values into the model (1)-(4).

Step 2. Run the model and retrieve the model output for tumor size and inflammatory cells per field in the same time instants as those in Table 1.

Step 3. Calculate the root mean square between the model output retrieved in Step 2 and the data in Table 1.

Model calibration has been made in stages, because it is not a continuous process due to the sudden extraction of the tumor with TURs and a calibration period is established for each operation. Besides, not all parameters are consid-

ered constant over the time. Immune response (whose related model parameters 


\begin{tabular}{clc}
\hline Parameter & Description & Term \\
\hline$k$ & Uninfected tumor cells growing rate. & $k T_{u}(t)$ \\
$\alpha$ & Production rate of inflammatory cells & $\alpha T_{u}(t)$ \\
& because of tumor cells. & $-\mu_{1} B(t)$ \\
$\mu_{1}$ & BCG natural death rate. & $-\mu_{2} E(t)$ \\
$\mu_{2}$ & Inflammatory cells natural death rate. \\
$p_{1}$ & BCG death rate because of inflammatory & $-p_{1} E(t) B(t)$ \\
& cells. & \\
$p_{2}$ & Tumor cell infection rate because of BCG. & $p_{2} T_{u}(t) B(t)$ \\
$p_{3}$ & $\begin{array}{l}\text { Infected tumor cells death rate because } \\
\text { of inflammatory cells. }\end{array}$ & $-p_{3} E(t) T_{i}(t)$ \\
$p_{4}$ & Inflammatory cells growth rate because & $p_{4} B(t) E(t)$ \\
& of BCG. & $-p_{5} E(t) T_{u}(t)$ \\
$p_{5}$ & $\begin{array}{l}\text { Inflammatory cells death rate because } \\
\text { of uninfected tumor cells. }\end{array}$ & $-p_{6} E(t) T_{u}(t)$ \\
$p_{6}$ & Tumor cell death rate because & \\
& of inflammatory cells (effectiveness). & \\
\hline
\end{tabular}

Table 2: Model parameters, description and modeling term. Positive terms mean growth of cells and negative terms mean death or removal of cells.

are the growing rate $p_{4}$ and the effectiveness rate $p_{6}$ ) is considered variable over the time.

For Patient $\mathrm{X}$ there are three different stages. The first stage starts the 01/Mar/2012 and lasts until 09/Aug/2012. In this first stage there are two different groups of parameters: the constant ones $\left(k, \alpha, \mu_{1}, \mu_{2}, p_{1}, p_{2}, p_{3}\right.$ and $\left.p_{5}\right)$ and the varying over the time ones $\left(p_{4}\right.$ and $\left.p_{6}\right)$ which are valid until the first BCG instillation occurs.

Together with the model parameters, the initial size of the tumor is unknown. Nevertheless, there is a diagnostic test made the 01/Mar/2012 where there is not any tumor evidence because the cystoscopy test is not positive if the tumor size is smaller than $5 \mathrm{~mm}$. However, according to medical criteria, the tumor size cannot be smaller than $3 \mathrm{~mm}$. Therefore, the initial size is also a parameter to be calibrated between the aforementioned values.

The second stage goes from 10/Aug/2012 to 25/May/2015. When the instillation is performed, the values of $p_{4}$ and $p_{6}$ change again. In this case, we do not know the value of the resulting tumor left inside the bladder after the instillations, therefore, this value is another parameter to be calibrated.

The third stage goes from 26/May/2015 to 26/Apr/2017. When the BCG instillation is performed, the values of $p_{4}$ and $p_{6}$ change again. The resulting tumor size after the TUR is also unknown and it is also calibrated.

For model calibration, we minimize function $F$ in stages, as described, using the rPSO algorithm [7]. 


\section{Results}

The calibrated parameter values for the Patient $\mathrm{X}$ are shown in Table 3 .

\begin{tabular}{cccc}
\hline Parameters & Value & Parameters & Value \\
\hline$k$ & 0.01563 & $T_{u}\left(T U R_{1}\right)$ & 0,02 \\
$\alpha$ & 0.005 & $p_{4}\left(T U R_{1}\right)$ & $1 \mathrm{e}-4$ \\
$\mu_{1}$ & 0.8 & $p_{6}\left(T U R_{1}\right)$ & $1.62 \mathrm{e}-5$ \\
$\mu_{2}$ & 0.0005 & & \\
$p_{1}$ & $8 \mathrm{e}-5$ & $T_{u}\left(T U R_{2}\right)$ & 0.02 \\
$p_{2}$ & $1 \mathrm{e}-3$ & $p_{4}\left(T U R_{2}\right)$ & $5.6 \mathrm{e}-4$ \\
$p_{3}$ & $1 \mathrm{e}-4$ & $p_{6}\left(T U R_{2}\right)$ & $6.3 \mathrm{e}-6$ \\
$p_{4}$ & 0.0 & & \\
$p_{5}$ & 0.0 & & \\
$p_{6}$ & 0.0 & & \\
$T_{u}(0)$ & 5.0 & & \\
$E(0)$ & 260 & & \\
\hline
\end{tabular}

Table 3: Model parameter values calibrated for Patient X. Observe that parameters $p_{4}$ and $p_{6}$ appear three times in the table and change over the time. Values accompanying $T U R_{1}$ and $T U R_{2}$ correspond to the variable parameters after the $T U R_{1}$ and $T U R_{2}$, respectively.

Parameter $p_{6}$ in the first stage is zero because the tumor cells growth parameter $k$ also includes the possible decrease because of the normal activity of the immune system. The following $p_{6}$ values obtained in the next stages reflects the increasing activity of the immune system because of BCG compared to the normal activity.

In Figure 3, the evolution of the tumor size and the immune system development are shown. In the lower figure (tumor size) the sudden drops correspond to TURs, when the tumor is removed. Then, it starts to grow again. Respect to the upper figure (immune response), we can see a quick increasing after TUR corresponding to the BCG instillations and how it influences the immune response. The saw teeth appearing when BCG is administered are due to the weekly doses of BCG instilled.

Due to the uncertainty in the future evolution, in order to predict the dynamics of the bladder cancer for Patient X, three different scenarios have been considered. In the favourable scenario the response of the immune system is greater than the second stage. The neutral scenario consists of the same response of the immune system as in the third stage, that is to say, the immune system response remains constant. The unfavourable scenario is based on the fact that the response of the immune system decreases in the same proportion as it decreased from the second to the third stage. In Table 4 the values of the parameters corresponding to the described scenarios are shown.

The day 0 in the figures correspond to $01 / \mathrm{Mar} / 2012$, coinciding with the first ultrasound test pointed out in Table 1. 

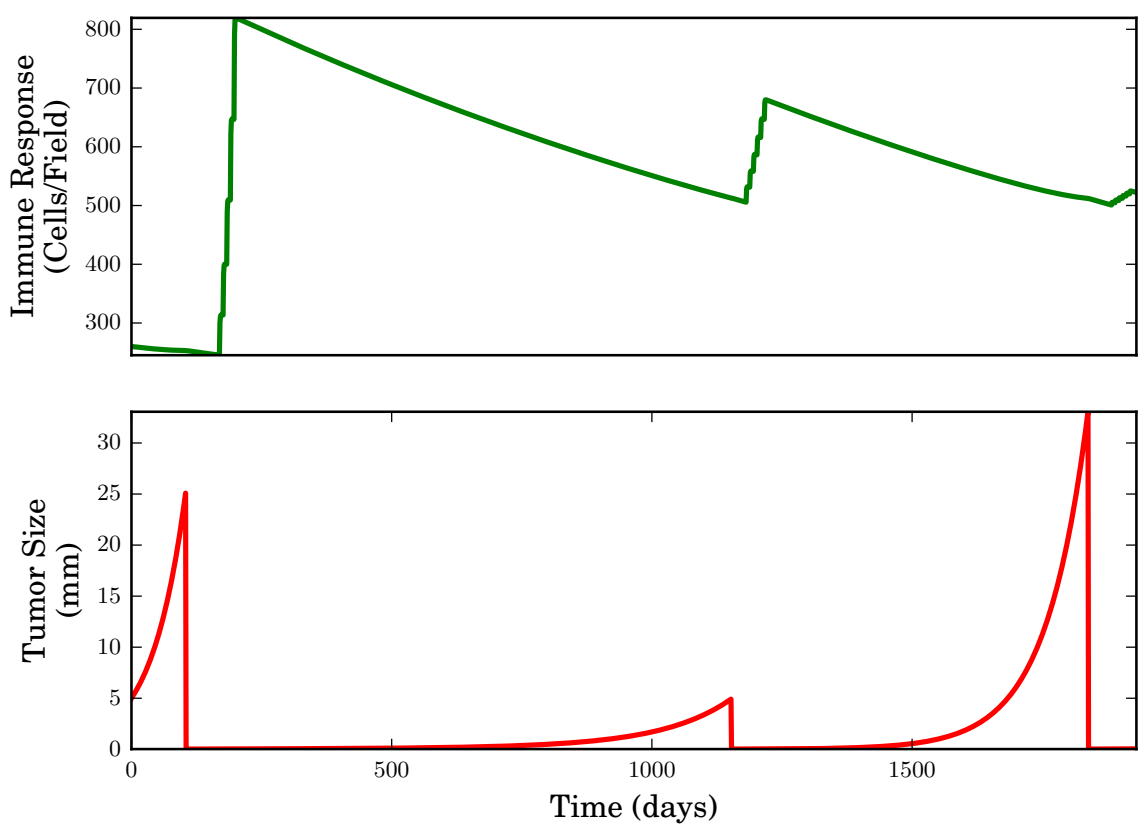

Figure 3: Here we show the graphs with the evolution of the immune response (upper) and the tumor size (lower) with the parameters obtained in the model calibration. The sudden drops in the lower graph correspond to the TURs, when tumors are removed. The sudden increasing in the upper graph correspond to the administration of the BCG. Time $t=0$ corresponds to 01/Mar/2012.

\begin{tabular}{cccc}
\hline Parameters & $\begin{array}{c}\text { Favourable } \\
\text { scenario }\end{array}$ & $\begin{array}{c}\text { Neutral } \\
\text { scenario }\end{array}$ & $\begin{array}{c}\text { Unfavourable } \\
\text { scenario }\end{array}$ \\
\hline$p_{4}$ & $1 \mathrm{e}-3$ & $5.6 \mathrm{e}-4$ & $1.14 \mathrm{e}-4$ \\
$p_{6}$ & $3.62 \mathrm{e}-5$ & $6.3 \mathrm{e}-6$ & $2.45 \mathrm{e}-6$ \\
Date tumor size $5 \mathrm{~mm}$ & No tumor & $15 / \mathrm{Aug} / 2018$ & $25 / \mathrm{May} / 2018$ \\
\hline
\end{tabular}

Table 4: Model parameter values in the three different scenarios: favourable, neutral and unfavourable.

In Figures 4, 5, 6, the prediction in the different scenarios are shown. The prediction starts in the dashed black vertical line. In the favourable scenario, the BCG treatment is successful and the tumor does not grow anymore after instillation treatment is finished. In the neutral scenario, bladder cancer recurrence with a tumor of size $5 \mathrm{~mm}$ is predicted for the $15 / \mathrm{Aug} / 2018$ and recurrence is expected. In the unfavourable scenario, bladder cancer recurrence with a tumor of size $5 \mathrm{~mm}$ is predicted for the 25/May/2018. 

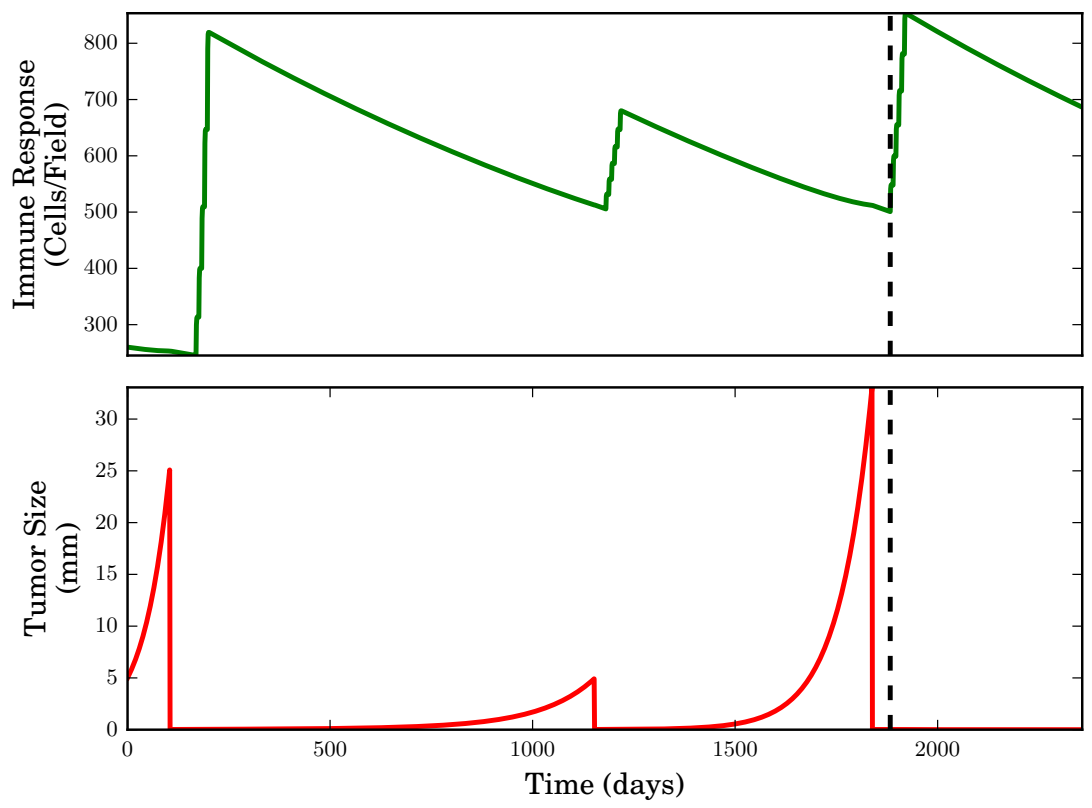

Figure 4: Favorable scenario. The prediction appears on the right of the dashed vertical black line. It can be seen that the tumor does not grow anymore, that is, the BCG treatment can kill the remainder tumor cells. Time $t=0$ corresponds to $01 / \mathrm{Mar} / 2012$.

\section{Conclusion}

In this paper, we present a dynamic mathematical model to describe the evolution of a bladder tumor in a given patient. The model is calibrated in several stages and the obtained parameters allowed us to give a prediction about the tumor growth over the next few months in three scenarios: favourable, neutral and unfavourable. To our knowledge, this is the first approach of this type applying the model to a given patient and the results seem promising.

Some predictions have been made and they will be checked in the near future. In fact, the Patient $\mathrm{X}$ will be called in the middle of June to measure the size of the tumor, if it exists. If the tumor is not present, the Patient $\mathrm{X}$ will be called in the middle of September for another medical revision.

When we have more data available, we will be able to check the validity of our approach, performing better and more accurate predictions, giving tools to the doctors to administer treatments, to schedule the patient's revisions and, in the future, to determine the best strategies to improve the patient's health avoiding tumor recurrences, if possible. 

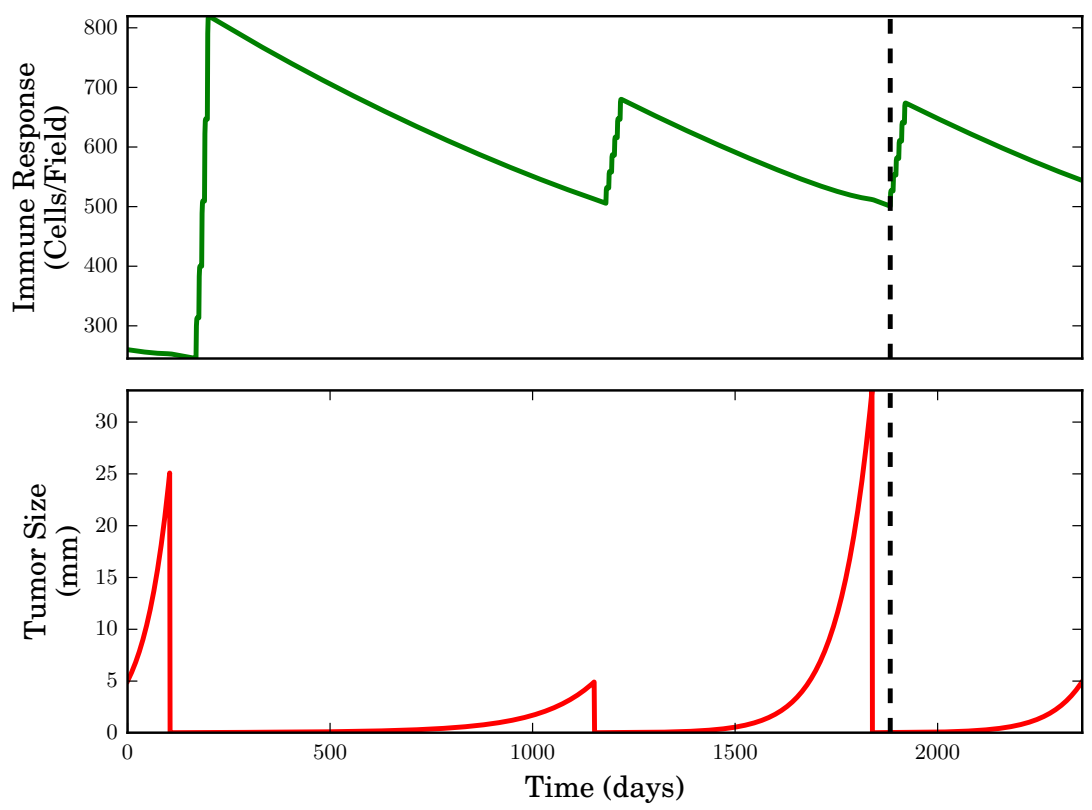

Figure 5: Neutral scenario. The prediction appears on the right of the dashed vertical black line. After a while, the tumor grows again and a recurrence is expected, reaching the size of $5 \mathrm{~mm}$ in the middle of August 2018. Time $t=0$ corresponds to $01 / \mathrm{Mar} / 2012$.

\section{References}

[1] Official Site for Hospital Universitari i Politècnic La Fe, Valencia, Spain. http://www.hospital-lafe.com. Accessed: 07/05/2018.

[2] Official Site for Spanish Medic Oncology Society. https://www.seom.org. Accessed: 08/05/2018.

[3] Svetlana Bunimovich-Mendrazitsky, Eliezer Shochat, and Lewi Stone. Mathematical model of BCG immunotherapy in superficial bladder cancer. Bulletin of Mathematical Biology, 69(6):1847-1870, apr 2007.

[4] R.W. Devere White and E. Stapp. Predicting prognosis in patients with superficial bladder cancer. ONCOLOGY, 12(12):1717-1723, 1998.

[5] R. T. Greenlee, M. B. Hill-Harmon, T. Murray, and M. Thun. Cancer statistics, 2001. CA: A Cancer Journal for Clinicians, 51(1):15-36, Jan 2001.

[6] Sten Holmang, Hans Hedelin, Claes Anderstrom, and Sonny L. Johansson. The relationship among multiple recurrences, progression and prognosis of 

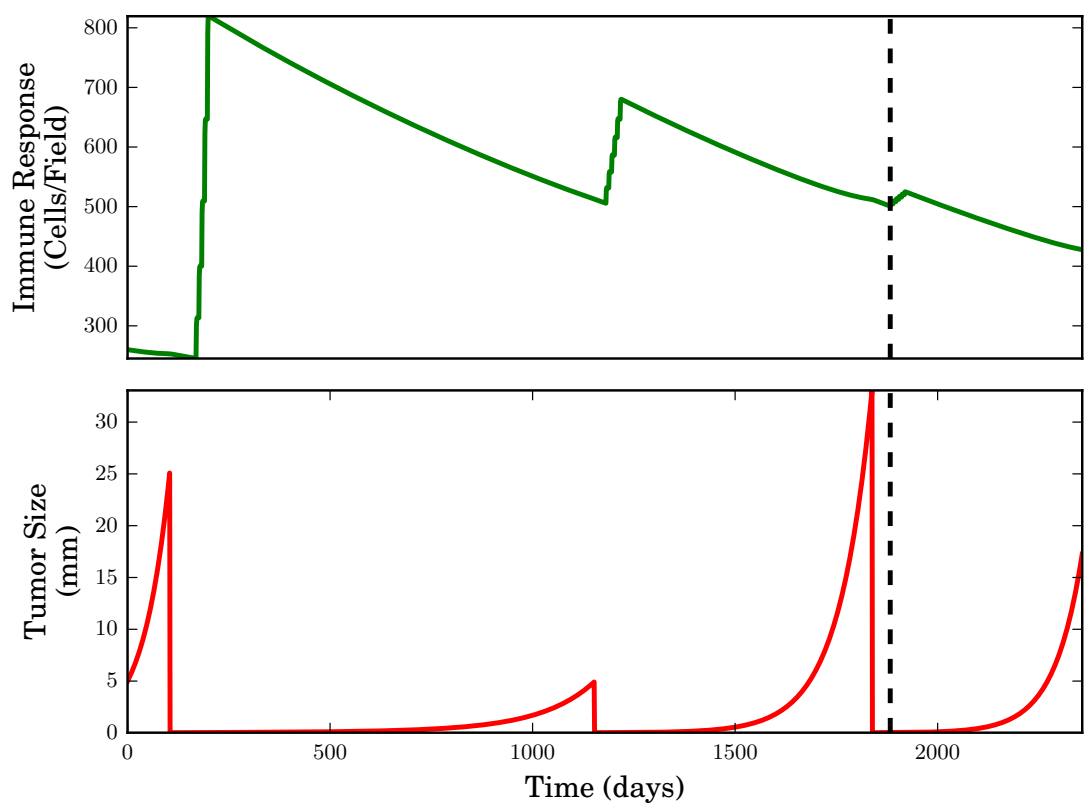

Figure 6: Unfavourable scenario. The prediction appears on the right of the dashed vertical black line. Here the effect of BCG is almost inexistent and the tumor grows quickly reaching the size of $5 \mathrm{~mm}$ at the end of May 2018. Time $t=0$ corresponds to $01 / \mathrm{Mar} / 2012$.

patients with stages TA and t1 transitional cell cancer of the bladder followed for at least 20 years. The Journal of Urology, 153(6):1823-1827, jun 1995.

[7] C. Jacob and N. Khemka. Particle swarm optimization in Mathematica. an exploration kit for evolutionary optimization. IMS'04, Proc. Sixth International Mathematica Symposium, Banff, Canada, 2004.

[8] Gil Redelman-Sidi, Michael S. Glickman, and Bernard H. Bochner. The mechanism of action of BCG therapy for bladder cancer - a current perspective. Nature Reviews Urology, 11(3):153-162, feb 2014.

[9] A.J. Wein, L.R. Kavoussi, A.C. Novick, A.W. Partin, and C.A. Peters. Campbell-Walsh Urology. Campbell-Walsh Urology. Elsevier Health Sciences, 2011. 